\title{
November 2013 Arizona Thoracic Society Notes
}

The November Arizona Thoracic Society meeting was held on Wednesday, 11/20/2013 at Shea Hospital beginning at 6:30 PM. There were 26 in attendance representing the pulmonary, critical care, sleep, nursing, radiology, and infectious disease communities.

As per the last meeting a separate area for upcoming meetings has been created in the upper left hand corner of the home page on the SWJPCC website.

A short presentation was made by Timothy Kuberski MD, Chief of Infectious Disease at Maricopa Medical Center, entitled "Clinical Evidence for Coccidioidomycosis as an Etiology for Sarcoidosis". Isaac Yourison, a medical student at the University of Arizona, will be working with Dr. Kuberski on his scholarly project. Mr. Yourison hypothesizes that certain patients diagnosed with sarcoidosis in Arizona really have coccidioidomycosis. It would be predicted that because of the immunosuppression, usually due to steroids, the sarcoidosis patients would eventually express the Coccidioides infection. The investigators will be collaborating with the University of Washington to perform polymerase chain reaction (PCR) on tissue samples diagnosed with sarcoidosis for Coccidioides.

There were 4 cases presented:

1. The first case was presented by Lewis Wesselius from the Mayo Clinic Arizona. The patient was a 56 year old woman with rheumatoid arthritis and a prior history of bronchiectasis. In 2009 she was diagnosed with Mycobacterium avium-intracellulare (MAI) on bronchoscopy and started on azithromycin, ethambutol, and rifabutin. She had been on etanercept which was held after her diagnosis of MAl. She had a negative sputum culture for MAI in September 2012 and her MAI medications were stopped. However, in May 2013 she had increasing symptoms and bronchoscopy demonstrated Pseudomonas and nontuberculous mycobacterium (NTB). She subsequently moved to Phoenix and a CT scan showed the size of her lung nodules to be increased. Bronchoscopic cultures showed Pseudomonas and Mycobacterium abscessus only sensitive to amikacin. She was treated with tigecycline and inhaled amikacin. A repeat CT scan indicated some decrease in size of lung nodules. Dr. Wesselius gave a short presentation on bronchiectasis associated with rheumatoid arthritis and NTB infection in these patients.

2. The second case was presented by Gerry Swartzberg. Dr. Schwartzberg showed a chest $x$-ray and asked the audience to guess the diagnosis. Jasminder Mand was the first to correctly guess allergic bronchopulmonary aspergillosis (ABPA) because of the finger in glove sign which best seen in the right upper lobe. The density forms from mucous impaction in a more central bronchus and has been referred to as a rabbit ear appearance, 
Mickey Mouse appearance, toothpaste shaped opacities, Y-shaped opacities, and V-shaped opacities. Dr. Mand also referred to this as the Churchill sign since it looks like the " $\mathrm{V}$ " gesture often associated with Churchill. The patient was begun on corticosteroids and a repeat chest $\mathrm{x}$-ray taken about a month later showed near clearing of the opacities.

3. Dr. Schwartzberg presented a second case of an elderly woman in her 80's with a history of bronchiectasis. Chest x-ray and CT scan showed several rapidly expanding lung masses. The radiographic appearance was not particularly suggestive of a diagnosis. There was a concern for malignancy and the majority thought bronchoscopy would be appropriate.

4. The last case was presented by Joshua Jewell, a third year pulmonary fellow in the Good Samaritan/VA program. The patient was a middle-aged man who had a history of diffusely metastatic hepatocellular cancer including to his lung and mediastinal lymph nodes. He was also diagnosed with sleep apnea and begun on continuous positive airway pressure (CPAP). He had increasing size of his neck and presented to the pulmonary clinic. Palpation revealed crepitus and a chest X-ray and CT scan confirmed the presence of subcutaneous air and a pneumomediastinum. Dr. Jewell hypothesized that the air was introduced or at least was exacerbated by the CPAP possibly from a ball valve mechanism. Most in the audience agreed this was a reasonable explanation but none had observed this phenomenon previously.

There being no further business the meeting was adjourned at about 8:30 PM. The next meeting is scheduled for Saturday, December 14, 8-12 AM in Tucson at the Kiewit Auditorium at the University of Arizona Medical Center. The next meeting in Phoenix will be held on Wednesday, January 22, 2014, 6:30 PM at Scottsdale Shea hospital.

Richard A. Robbins, M.D. 